\title{
Searches for lepton flavour and number violation in $\mathrm{K}^{+}$ and $\pi^{0}$ decays at the NA62 experiment
}

\author{
Elisa Minucci ${ }^{a, 1, *}$ \\ ${ }^{a}$ CERN-European Organization for Nuclear Research, \\ Esplanade des particules 1 Ch-1211, Geneva 23,Switzerland \\ E-mail: elisa.minucci@cern.ch
}

The NA62 experiment at CERN collected a large sample of charged kaon decays into final states with multiple charged particles in 2017-2018. Results from searches for lepton flavour and number violating decays in $K^{+}$and $\pi^{0}$ to final states containing a lepton pair, based on this data set, are presented, reaching sensitivities of $O\left(10^{-11}-10^{-10}\right)$.

*** The European Physical Society Conference on High Energy Physics (EPS-HEP2021), ***

*** 26-30 July $2021 * * *$

*** Online conference, jointly organized by Universität Hamburg and the research center DESY ***

\footnotetext{
${ }^{1}$ Supported by ERC-STG802836 (AxScale)

* Speaker, for the NA62 Collaboration: A. Akmete, R. Aliberti, F. Ambrosino, R. Ammendola, B. Angelucci, A.
} Antonelli, G. Anzivino, R. Arcidiacono, T. Bache, A. Baeva, D. Baigarashev, L. Bandiera, M. Barbanera, J. Bernhard, A. Biagioni, L. Bician, C. Biino, A. Bizzeti, T. Blazek, B. Bloch-Devaux, P. Boboc, V. Bonaiuto, M. Boretto, M. Bragadireanu, A. BrianoOlvera, D. Britton, F. Brizioli, M.B. Brunetti, D. Bryman, F. Bucci, T. Capussela, J. Carmignani, A. Ceccucci, P. Cenci, V. Cerny, C. Cerri, B.Checcucci, A. Conovaloff, P. Cooper, E. Cortina Gil, M. Corvino, F. Costantini, A. Cotta Ramusino, D. Coward, G. D’Agostini, J. Dainton, P. Dalpiaz, H. Danielsson, M. D’Errico, N. De Simone, D. DiFilippo, L. DiLella, N. Doble, B. Dobrich, F. Duval, V. Duk, D. Emelyanov, J. Engelfried, T. Enik, N. Estrada-Tristan, V. Falaleev, R. Fantechi, V. Fascianelli, L. Federici, S. Fedotov, A. Filippi, R. Fiorenza, M. Fiorini, J. Fry, J. Fu, A. Fucci, L. Fulton, E. Gamberini, L. Gatignon, G. Georgiev, S. Ghinescu, A. Gianoli, M. Giorgi, S. Giudici, F. Gonnella, K. Gorshanov, E. Goudzovski, C. Graham, R. Guida, E. Gushchin, F. Hahn, H. Heath, J. Henshaw, Z. Hives, E.B. Holzer, T. Husek, O. Hutanu, D. Hutchcroft, L. Iacobuzio, E. Iacopini, E. Imbergamo, B. Jenninger, J. Jerhot, R.W. Jones, K. Kampf, V. Kekelidze, D. Kerebay, S. Kholodenko, G. Khoriauli, A. Khotyantsev, A. Kleimenova, A. Korotkova, M. Koval, V. Kozhuharov, Z. Kucerova, Y. Kudenko, J. Kunze, V. Kurochka, V. Kurshetsov, G. Lanfranchi, G. Lamanna, E. Lari, G. Latino, P. Laycock, C. Lazzeroni, M. Lenti, G. LehmannMiotto, E. Leonardi, P. Lichard, L. Litov, P. LoChiatto, R. Lollini, D. Lomidze, A. Lonardo, P. Lubrano, M. Lupi, N. Lurkin, D. Madigozhin, I. Mannelli, A. Mapelli, F. Marchetto, R.Marchevski, S. Martellotti, P. Massarotti, K. Massri, E.Maurice, A. Mazzolari, M. Medvedeva, A. Mefodev, E. Menichetti, E. Migliore, E.Minucci, M. Mirra, M. Misheva, N. Molokanova, M. Moulson, S. Movchan, M. Napolitano, I. Neri, F. Newson, A. Norton, M. Noy, T. Numao, V. Obraztsov, A. Okhotnikov, A. Ostankov, S. Padolski, R. Page, V. Palladino, I. Panichi, A. Parenti, C. Parkinson, E. Pedreschi, M. Pepe, M. Perrin-Terrin, L.Peruzzo, P. Petrov, Y. Petrov, F. Petrucci, R. Piandani, M. Piccini, J. Pinzino, I. Polenkevich, L. Pontisso, Yu. Potrebenikov, D. Protopopescu, M. Raggi, M. ReyesSantos, M. Romagnoni, A. Romano, P. Rubin, G. Ruggiero, V. Ryjov, A. Sadovsky, A. Salamon, C. Santoni, G. Saracino, F. Sargeni, S. Schuchmann, V. Semenov, A. Sergi, A. Shaikhiev, S. Shkarovskiy, M. Soldani, D. Soldi, M. Sozzi, T. Spadaro, F. Spinella, A. Sturgess, V. Sugonyaev, J. Swallow, A. Sytov, G. Tinti, A. Tomczak, S. Trilov, P. Valente, B. Velghe, S. Venditti, P. Vicini, R.Volpe, M. Vormstein, H. Wahl, R. Wanke, V. Wong, B. Wrona, O. Yushchenko, M. Zamkovsky, A. Zinchenko. 


\section{Introduction}

Lepton Number (LN) and Lepton Flavour number (LF) are conserved quantum number in the Standard Model (SM), although their conservation is not imposed by any local gauge symmetry. Searches for LN and LF violation are powerful probes to search for physics beyond the SM, exploring high mass scales. Several new physics models build to address experimental observations not explained by the SM, naturally introduce LN or LF violation. Examples are the see-saw mechanism [1], which provides a source of $\mathrm{LN}$ violation via the exchange of a Majorana neutrino; SM extensions with an additional $\mathrm{U}(1)$ gauge symmetry and an associated $\mathrm{Z}$ ' boson [2, 3], SM extensions with light pseudoscalar bosons [4], or leptoquarks models [5] can be source of LF violation. Previous limits, at $90 \% \mathrm{CL}$, on $\mathrm{LN}$ and LF violating $K^{+}$and $\pi^{0}$ decays are: $K^{+} \rightarrow$ $\pi^{-} \mu^{+} \mu^{+}<4.2 \times 10^{-11}, K^{+} \rightarrow \pi^{-} e^{+} e^{+}<2.2 \times 10^{-10}[6], K^{+} \rightarrow \pi^{-} \mu^{+} e^{+}<5.0 \times 10^{-10}, K^{+} \rightarrow$ $\pi^{+} \mu^{-} e^{+}<5.2 \times 10^{-11}$ [7], $K^{+} \rightarrow \pi^{+} \mu^{+} e^{-}<1.3 \times 10^{-11}[8], \pi^{0} \rightarrow \mu^{-} e^{+}<3.4 \times 10^{-9}$ [7] and $\pi^{0} \rightarrow \mu^{+} e^{-}<3.8 \times 10^{-10}[9]$.

In this work searches are presented for LN violation decay $K^{+} \rightarrow \pi^{-} \mu^{+} e^{+}\left(\pi^{-}\right.$channel) and LF violation decays $K^{+} \rightarrow \pi^{+} \mu^{-} e^{+}$( $\mu^{-}$channel) and $\pi^{0} \rightarrow \mu^{-} e^{+}$. These searches have been performed at the NA62 experiment at the CERN super proton syncrotron (SPS), using the data collected in 2017 and 2018.

The main goal of the experiment is the measurement of the $K^{+} \rightarrow \pi^{+} v \bar{v}$ branching ratio, theoretically well predicted in the SM, $B r_{S M}=(8.3 \pm 1) \times 10^{-11}$ [10]. This is one of the golden mode to search for new physics effects: any deviation from the SM branching ratio would be an hint of new physics.

The NA62 beam line and detector are described in detail in [11]. The NA62 beam is produced by the interaction of a $400 \mathrm{GeV} / c$ proton beam, coming from the CERN SPS, with a beryllium target. It is composed of positive charged particles of which $6 \%$ are kaons and it has a central momentum of $75 \mathrm{GeV} / \mathrm{c}$ with a momentum bite of $\Delta p / p=1 \%$. The kaons in the beam are identified by a Cherenkov counter detector, KTAG. Before entering the vacuum region downstream, the kaon momentum and direction are measured by the Gigatracker (GTK), composed of three silicon pixel stations of $6 \times 3 \mathrm{~cm}^{2}$ surface area exposed to the full beam rate. The momenta and directions of charged particles produced in $\mathrm{K}^{+}$decays in a $75 \mathrm{~m}$ long fiducial volume (FV) are measured by a magnetic spectrometer (STRAW). Particle identification is provided by a ring-imaging Cherenkov detector (RICH), a quasi-homogeneous liquid krypton electromagnetic calorimeter(LKr), hadronic calorimeters (MUV1,2) and a muon detector (MUV3). Photons are detected by an hermetic photon veto system covering angles up to $50 \mathrm{mrad}$ from the beam axis, composed of twelve annular lead glass detectors (LAV1-12), two lead/scintillator sampling calorimeters (IRC, SAC) close to the beam axis and the LKr. The RICH provides a trigger time with 70 ps precision. Two scintillator hodoscopes, NA48-CHOD and CHOD, each arranged in four quadrants, provide trigger signals and time measurements for downstream charged particles with $200 \mathrm{ps}$ and 800 ps precision, respectively.

\section{Analysis}

Searches for LN and LF violation decays are performed following a blind analysis strategy. The branching ratios for the signal decays are measured relative to the normalisation channel 
$K^{+} \rightarrow \pi^{+} \pi^{+} \pi^{-}\left(K_{3 \pi}\right)$. A three-track final state selection is common to signals and nomalisation. It requires three tracks reconstructed in the STRAW within the geometrical acceptance of the downstream detectors used in the analysis, within $5 \mathrm{~ns}$ of the trigger time and forming a vertex of total charge +1 with a longitudinal distance from the target $105<\mathrm{Z}_{\mathrm{vtx}}<180 \mathrm{~m}$. To ensure that the event is associated to a kaon decay the vertex time, defined as the weighted mean of the track times, is required to be within $3 \mathrm{~ns}$ of the time of the reconstructed $K^{+}$in the KTAG detector. Events with LAV signals within $3 \mathrm{~ns}$ of the trigger time are rejected, providing a photon veto. The total vertex three-momentum must have a magnitude within $2.5 \mathrm{GeV} / \mathrm{c}$ of the mean beam momentum, and the transverse momentum with respect to the beam axis should be $\mathrm{p}_{\mathrm{T}}<35 \mathrm{MeV} / \mathrm{c}$.

For the normalisation channel selection, the three-track invariant mass reconstructed under the $3 \pi$ mass hypothesis is required to be consistent with the charged kaon mass within $3 \sigma_{3 \pi}$, where the measured mass resolution is $\sigma_{3 \pi}=0.9 \mathrm{MeV} / \mathrm{c} 2$.

For the signal selection particle identification (PID) conditions are applied, using the ratio $E / p$ calculated from the energy $(E)$ of the associated $\mathrm{LKr}$ cluster and its momentum $(p)$ and the information from the muon detector MUV3 to isolate candidate $\pi^{\mp} \mu^{ \pm} e^{+}$final states. The range of the vertex longitudinal position is optimised to reduce the background from $\mathrm{K}^{+}$decays upstream of the FV. It is required that $Z_{\mathrm{vtx}}>107(111) \mathrm{m}$ for the $\pi^{-}\left(\mu^{-}\right)$channel. The kinematic variable used to distinguish between signal and background is the invariant mass of the three selected tracks, built under the $\pi-\mu-e$ mass hypothesis, $m_{\pi \mu e}$. The $m_{\pi \mu e}$ region close to the charged kaon mass, $478-510 \mathrm{MeV} / \mathrm{c}^{2}$, kept masked during the analysis, includes the signal region, $490-498 \mathrm{MeV} / \mathrm{c}^{2}$ and two control regions. The $m_{\pi \mu e}$ resolution, obtained from simulation is $1.4 \mathrm{MeV} / \mathrm{c}^{2}$. The search for $K^{+} \rightarrow \pi^{+} \pi^{0}$ followed by $\pi^{0} \rightarrow \mu^{-} e^{+}$, is performed on the sample of events passing the $\mu^{-}$channel selection by requiring that the reconstructed mass of the $\mu-e$ pair is consistent with the $\pi^{0}$ mass.

\section{Trigger and data sample}

The NA62 trigger system is composed of a hardware level (L0) and a software level (L1) [12]. For the analyses reported here, three dedicated trigger chains are implemented to collect kaon decays with multiple charged particles in the final state, running concurrently with the trigger chain dedicated to the main goal of the experiment: the multi-track (MT), electron multi-track (eMT), and muon multi-track $(\mu \mathrm{MT})$ triggers. The L0 MT trigger requires a signal in the RICH and coincident signals in two opposite CHOD quadrants. The $\mu \mathrm{MT}$ select events with at least one muon in the final state, requiring in addition to the MT condition that there is a signal in the MUV3 and a minimum energy deposit of $10 \mathrm{GeV}$ in the $\mathrm{LKr}$ (LKr10), while the eMT chain selects events enriched with electrons, by additionally requiring a minimum energy deposit of $20 \mathrm{GeV}$ in the $\mathrm{LKr}$ (LKr20). Events collected by the L0 triggers are further filtered by a L1 trigger, selecting events with a $K^{+}$ identified by the KTAG within $5 \mathrm{~ns}$ of the trigger time and a negative track reconstructed in the STRAW. For most of the data sample the L1 $\mu \mathrm{MT}$ trigger also requires fewer than 3 signals in total in LAV stations $2-11$ within $6 \mathrm{~ns}$ of the trigger time. Tipical downscale factors for the MT, $\mu \mathrm{MT}$, and eMT trigger chains are: $D_{M T}=100, D_{\mu M T}=8$, and $D_{e M T}=8$.

Data collected with a minimum bias trigger, are used for particle identification and trigger efficiency studies. 

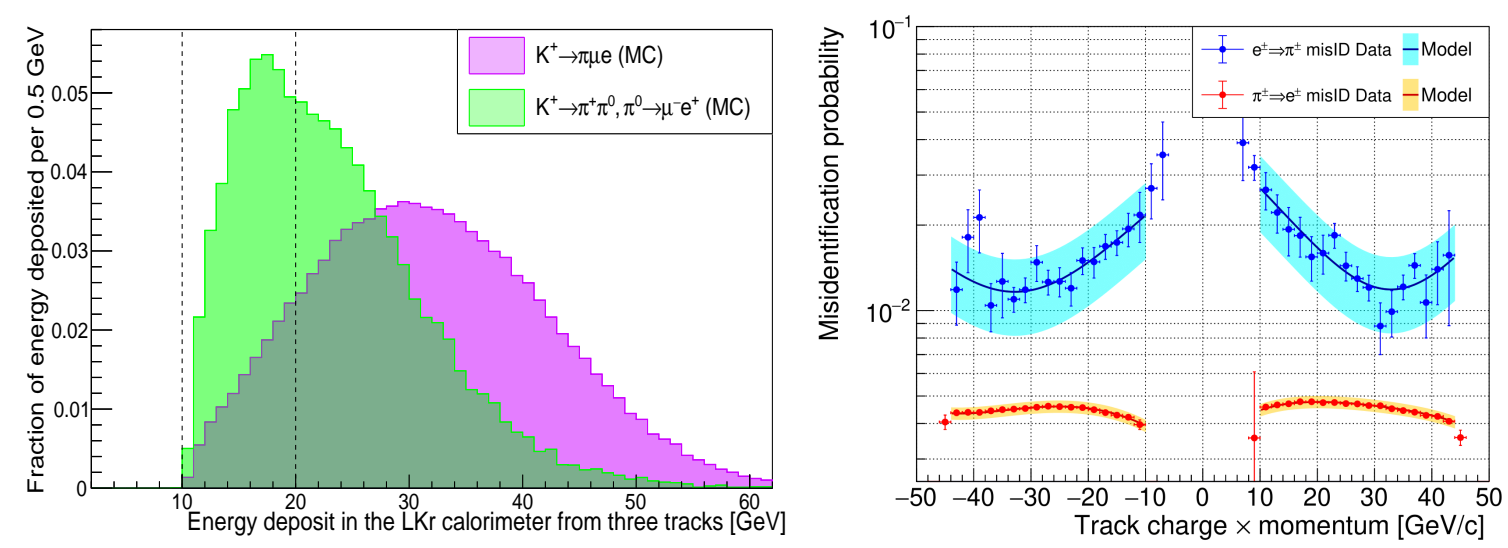

Figure 1: Left: Distributions of energy deposited in the LKr associated with the three selected STRAW tracks for events passing the signal selection, $K^{+} \rightarrow \pi^{ \pm} \mu^{\mp} e^{+}$and $K^{+} \rightarrow \pi^{+} \pi^{0}$ followed by $\pi^{0} \rightarrow \mu^{-} e^{+}$, obtained from MC simulations after data-driven corrections to the energy of the $\mathrm{LKr}$ pion cluster. Right: Misidentification probabilities of $\pi^{ \pm}$as $e^{ \pm}$(blue) and of $e^{ \pm}$as $\pi^{ \pm}$(red) as a function of the track charged times momentum.

The measured efficiency of the MT trigger for normalisation events is $\epsilon_{\mathrm{n}}=(93.2 \pm 0.5) \times 10^{2}$, and the result for signal-like events is consistent with $\epsilon_{\mathrm{n}}$ within $1 \%$. The L0 MUV3 and L1 LAV conditions in the $\mu M T$ trigger have negligible inefficiency for signal-like events since similar conditions are applied offline in the selection. The efficiencies of the LKr10 and LKr20 conditions depend on the total energy deposited in the LKr (Figure 1 left).

The effective number of $K^{+}$decays in the FV is:

$$
N_{K}=\sum_{i} N_{K}^{i}=\frac{1}{\mathcal{B}\left(K_{3 \pi}\right) A_{\mathrm{n}} \varepsilon_{\mathrm{n}}} \cdot \sum_{i}\left(N_{3 \pi}^{i} \frac{D_{\mathrm{MT}}^{i}}{D_{\mathrm{eff}}^{i}}\right)=(1.33 \pm 0.02) \times 10^{12},
$$

where the index $i$ runs over different data-taking periods, $N_{3 \pi}^{i}$ are the numbers of normalisation $K_{3 \pi}$ events selected with the MT trigger with downscaling factor $D_{\mathrm{MT}}^{i}$, and $D_{\text {eff }}^{i}$ are the effective downscaling factors of the three signal trigger chains. In Eq. $1, \mathcal{B}\left(K_{3 \pi}\right)=(5.583 \pm 0.024) \times 10^{2}$ and $A_{\mathrm{n}}=10.18 \times 10^{-2}$ are the branching ratio and selection acceptance of the $K_{3 \pi}$ decay. The quoted uncertainty in $N_{K}$ accounts for any inaccuracy in the description of the beam momentum spectrum and STRAW inefficiency in simulations.

\section{Background}

Backgrounds arise from $K^{+}$decays together with the decay in flight (DIF) of one or more particles in the final state and particle misidentification. Among the decay in flight the most frequent one is the $\pi^{ \pm} \rightarrow \mu^{ \pm} v$. The $\pi^{ \pm} \rightleftharpoons e^{ \pm}$misidentification probabilities, derived from the $\mathrm{E} / \mathrm{p}$ measurement, are evaluated using $K^{+} \rightarrow \pi^{+} \pi^{+} \pi^{-}$and $K^{+} \rightarrow \pi^{+}\left[e^{+} e^{-} \gamma\right]_{\pi^{0}}$ events and are shown in Figure 1 on the righthand side plot. The misidentification probabilities of $\pi^{ \pm}$and $e^{ \pm}$as $\mu^{ \pm}$due to the presence of accidental muons in the MUV3 detector are also considered.

The estimation of the background in the signal region is done using simulations. Data driven corrections are applied as a weight taking into account trigger efficiency effects, observed differences 
Table 1: Summary of inputs to the single event sensitivity calculation and corresponding resulting values for each search. The signal acceptances, $A_{\mathrm{s}}$, are displayed with statistical uncertainties only; other uncertainties quoted are quadratic sums of the statistical and systematic uncertainties.

\begin{tabular}{|c|c|c|l|}
\hline & $K^{+} \rightarrow \pi^{-} \mu^{+} e^{+}$ & $K^{+} \rightarrow \pi^{+} \mu^{-} e^{+}$ & $\pi^{0} \rightarrow \mu^{-} e^{+}$ \\
\hline$A_{\mathrm{S}} \times 10^{2}$ & $4.90 \pm 0.02$ & $6.21 \pm 0.02$ & $3.11 \pm 0.02$ \\
$\varepsilon_{\mathrm{LKr} 10} \times 10^{2}$ & $97.5 \pm 1.3$ & $97.5 \pm 1.3$ & $92.9 \pm 1.2$ \\
$\varepsilon_{\mathrm{LKr} 20} \times 10^{2}$ & $74.1 \pm 1.6$ & $73.3 \pm 1.6$ & $45.3 \pm 1.0$ \\
\hline \hline $\mathcal{B}_{\mathrm{SES}} \times 10^{11}$ & $1.82 \pm 0.08$ & $1.44 \pm 0.05$ & $13.9 \pm 0.9$ \\
\hline
\end{tabular}

Table 2: Pedicted numbers of background events and observed number of events in signal regions. New limits at $90 \% \mathrm{CL}$ on the branching ratio of each signal channels have been set using the $\mathrm{CL}_{s}$ method.

\begin{tabular}{|c|c|c|c|}
\hline & $n_{b g}$ & $n_{\text {obs }}$ & NA62 limits $(90 \%$ CL $)$ \\
\hline$K^{+} \rightarrow \pi^{-} \mu^{+} e^{+}$ & $1.07 \pm 0.20$ & 0 & $\mathcal{B}\left(K^{+} \rightarrow \pi^{-} \mu^{+} e^{+}\right)<4.2 \times 10^{-11}$ \\
$K^{+} \rightarrow \pi^{+} \mu^{-} e^{+}$ & $0.92 \pm 0.34$ & 2 & $\mathcal{B}\left(K^{+} \rightarrow \pi^{+} \mu^{-} e^{+}\right)<6.6 \times 10^{-11}$ \\
$\pi^{0} \rightarrow \mu^{-} e^{+}$ & $0.23 \pm 0.15$ & 0 & $\mathcal{B}\left(\pi^{0} \rightarrow \mu^{-} e^{+}\right)<3.2 \times 10^{-10}$ \\
\hline
\end{tabular}

between data and simulation such as the energy deposited by pions in the $\mathrm{LKr}$ and misindentification probabilities.

\section{Results}

The single event sensitivities, $\mathcal{B}_{S E S}$, defined for each process as the branching ratios corresponding to the observation of one signal event, are computed as:

$$
\mathcal{B}_{\mathrm{SES}}=\left[\sum_{i}\left(\mathcal{B}_{\mathrm{SES}}^{i}\right)^{-1}\right]^{-1}, \quad \mathcal{B}_{\mathrm{SES}}^{i}=\frac{1}{N_{K}^{i} A_{\mathrm{s}} \varepsilon_{\mathrm{S}}^{i}}=\mathcal{B}\left(K_{3 \pi}\right) \frac{A_{\mathrm{n}} D_{\mathrm{eff}}^{i}}{A_{\mathrm{S}} N_{3 \pi}^{i} D_{\mathrm{MT}}^{i}} \frac{\varepsilon_{\mathrm{n}}}{\varepsilon_{\mathrm{S}}^{i}},
$$

where $A_{\mathrm{s}}$ are the signal acceptances, and $\varepsilon_{\mathrm{s}}^{i}$ are the trigger efficiencies for signal events. Efficiencies for trigger components present in both normalisation and signal trigger chains cancel to $1 \%$ precision, except for the $\operatorname{LKr} 10(20)$ components $\left(\varepsilon_{\mathrm{LKr} 10(20)}\right)$, which depend on the energy deposited in the $\mathrm{LKr}$ and are not present in the MT trigger chain. For the $\pi^{0} \rightarrow \mu^{-} e^{+}$search, $\mathcal{B}_{\mathrm{SES}}^{i}$ is divided by $\mathcal{B}\left(K^{+} \rightarrow \pi^{+} \pi^{0}\right)=(20.67 \pm 0.08) \times 10^{-2}$. A summary of inputs to the single event sensitivity calculation and the $\mathcal{B}_{\text {SES }}$ for the full data set and for each signal channel are given in Table 1.

After the validation of the background estimation in control regions, the blinded regions have been opened. The invariant mass distributions are shown in Figure 2. The observed numbers of events in the signal regions are in good agreement and new limits on the branching ratios at $90 \%$ CL for each signal channel have been set and are reported in Table 2.

\section{Conclusion}

The new limits reported here for the $K^{+} \rightarrow \pi^{-} \mu^{+} e^{+}, K^{+} \rightarrow \pi^{+} \mu^{-} e^{+}$and $\pi^{0} \rightarrow \mu^{-} e^{+}$represent an improvement by one order of magnitude over previous limits. The detailed description of the 

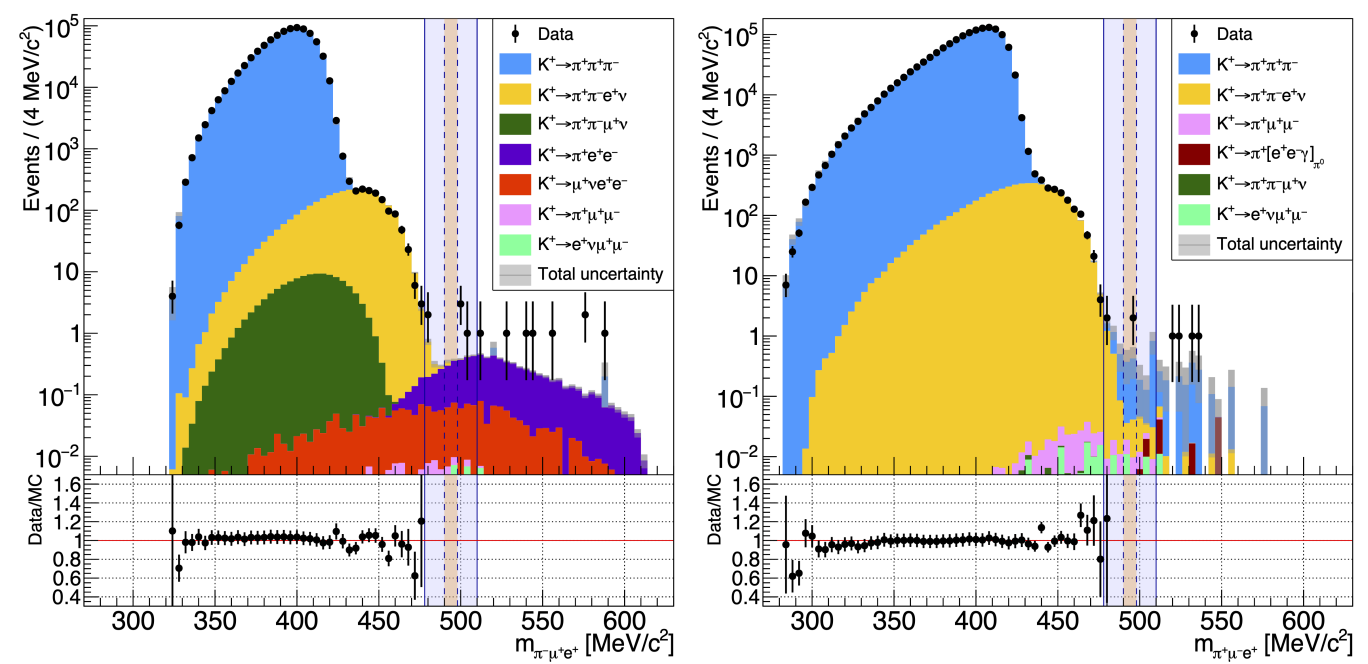

Figure 2: $K^{+} \rightarrow \pi^{-} \mu^{+} e^{+}$(left) and $K^{+} \rightarrow \pi^{+} \mu^{-} e^{+}$(right) invariant mass distribution

analysis is reported in [13]. NA62 resumes data-taking in 2021, with higher beam intensity and new detectors, and there will be the possibility of collecting additional data to study LN and LF violating decays.

\section{References}

[1] P. Minkowski, Phys. Lett. B 67 (1977) 421.

[2] P. Langacker, Phys. Atom. Nucl. 68 (2005) 1190.

[3] P. Langacker, Rev. Mod. Phys. 81 (2009) 3.

[4] M. Bordone et al., J. High Energy Phys. 1810 (2018) 148.

[5] J.C. Pati, A. Salam, Phys. Rev. D 10 (1974) 27.

[6] E. Cortina Gil et al., Phys. Lett. B 797 (2019) 134794.

[7] R. Appel et al., Phys. Rev. Lett. 85 (2000) 2877.

[8] A. Sheret al., Phys. Rev. D72 (2005) 012005.

[9] R. Appel et al., Phys. Rev. Lett. 85 (2000) 2450

[10] A. J. Buras, D. Buttazzo, R. Knegjens, JHEP 1511, 166 (2015)

[11] E. Cortina Gil et al., JINST 12 (2017) P05025.

[12] R. Ammendola et al., Nucl. Instr. Meth. A929 (2019) 1.

[13] R. Aliberti et al., Phys. Rev. Lett. 127 (2021) 131802. 